Supplement of Atmos. Chem. Phys., 20, 4969-4986, 2020

https://doi.org/10.5194/acp-20-4969-2020-supplement

(c) Author(s) 2020. This work is distributed under

the Creative Commons Attribution 4.0 License.

(c) (i)

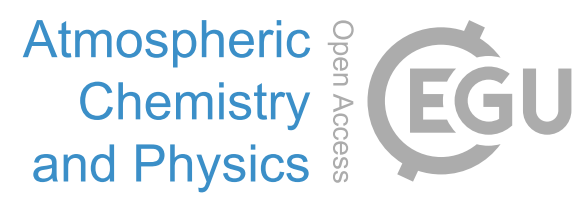

Supplement of

\title{
Linkage between dust cycle and loess of the Last Glacial Maximum in Europe
}

Erik Jan Schaffernicht et al.

Correspondence to: Erik Jan Schaffernicht (erik.research@eclipso.ch)

The copyright of individual parts of the supplement might differ from the CC BY 4.0 License. 
Table S1: Loess mass accumulation rates; encompassing all particle sizes (MAR) or only particles with less than $10 \mu \mathrm{m}$ diameter (MAR10). Reconstructed from fieldwork samples dated to the LGM or to a period encompassing the LGM. Proportion of particles smaller than $10 \mu \mathrm{m}$ in diameter (Frac10). MAR ranges itemized by their limits. For sites with more than one reconstructed MAR each rate is shown separately. Missing value (0.00); country codes (CC) conform with ISO 3166.

\begin{tabular}{|c|c|c|c|c|c|c|}
\hline Site & $\mathrm{CC}$ & $\begin{array}{r}\text { Lat } \\
{ }^{\circ} \mathrm{N}\end{array}$ & $\begin{array}{r}\text { Lon } \\
{ }^{\circ} \mathrm{E}\end{array}$ & $\begin{array}{r}\text { MAR } \\
\mathrm{g} \mathrm{m}^{-2} \mathrm{yr}^{-1}\end{array}$ & $\begin{array}{l}\text { MAR10 } \\
\mathrm{g} \mathrm{m}^{-2} \mathrm{yr}^{-1}\end{array}$ & Frac10 \\
\hline Achenheim [1] & FR & 48.35 & 7.38 & 331.00 & 82.75 & 0.25 \\
\hline Achenheim [2] & FR & 48.35 & 7.38 & 257.00 & 0.00 & 0 \\
\hline Albertirsa [3] & $\mathrm{HU}$ & 47.26 & 19.64 & 386.00 & 96.50 & 0.25 \\
\hline Albertirsa [2] & $\mathrm{HU}$ & 47.26 & 19.64 & 841.00 & 0.00 & 0 \\
\hline Albertirsa [4] & $\mathrm{HU}$ & 47.26 & 19.64 & 587.00 & 134.00 & 0.23 \\
\hline Am Bingert Wiesbaden [2] & $\mathrm{DE}$ & 50.12 & 8.28 & 361.00 & 0.00 & 0 \\
\hline Arapovychi (Arapovichi) [5] & UA & 51.95 & 33.31 & 666.00 & 166.50 & 0.25 \\
\hline Basaharc [4] & $\mathrm{HU}$ & 47.80 & 18.84 & 348.00 & 79.00 & 0.23 \\
\hline Batajnica [4] & RS & 44.92 & 20.32 & 329.00 & 75.00 & 0.23 \\
\hline Böckingen [2] & $\mathrm{DE}$ & 49.13 & 9.18 & 3300.00 & 0.00 & 0 \\
\hline Bodrogkeresztúr 1 [4] & $\mathrm{HU}$ & 48.13 & 21.40 & 381.00 & 87.00 & 0.23 \\
\hline Bönnigheim A [2] & $\mathrm{DE}$ & 49.04 & 9.14 & 131.00 & 0.00 & 0 \\
\hline Bönnigheim B [6] & $\mathrm{DE}$ & 49.04 & 9.14 & 242.00 & 60.50 & 0.25 \\
\hline Bönnigheim B [2] & $\mathrm{DE}$ & 49.04 & 9.14 & 283.00 & 0.00 & 0 \\
\hline Bönnigheim B [2] & $\mathrm{DE}$ & 49.04 & 9.14 & 1500.00 & 0.00 & 0 \\
\hline Bryansk [5] & $\mathrm{RU}$ & 53.15 & 34.06 & 394.00 & 98.50 & 0.25 \\
\hline Crvenka [4] & RS & 45.66 & 19.48 & 197.00 & 45.00 & 0.23 \\
\hline Csorgókút 1 [4] & $\mathrm{HU}$ & 48.13 & 21.40 & 284.00 & 65.00 & 0.23 \\
\hline Csorgókút 2 [4] & $\mathrm{HU}$ & 48.13 & 21.40 & 453.00 & 103.00 & 0.23 \\
\hline Debrecen (Alföldi brickyard) [4] & $\mathrm{HU}$ & 47.53 & 21.57 & 237.00 & 54.00 & 0.23 \\
\hline Dolní Vestonice [6] & $\mathrm{CZ}$ & 48.89 & 16.66 & 758.00 & 189.50 & 0.25 \\
\hline Dolní Vĕstonice [2] & $\mathrm{CZ}$ & 48.89 & 16.66 & 754.00 & 0.00 & 0 \\
\hline Dolní Vĕstonice [2] & $\mathrm{CZ}$ & 48.89 & 16.66 & 1100.00 & 0.00 & 0 \\
\hline Dunaszekcső[4] & $\mathrm{HU}$ & 46.07 & 18.75 & 707.00 & 161.00 & 0.23 \\
\hline Dunaújváros [4] & $\mathrm{HU}$ & 46.97 & 18.94 & 1238.00 & 282.00 & 0.23 \\
\hline Dybawka [7] & $\mathrm{PL}$ & 49.79 & 22.69 & 1195.00 & 0.00 & 0 \\
\hline Erdut [4] & $\mathrm{HR}$ & 45.53 & 19.06 & 215.00 & 49.00 & 0.23 \\
\hline Gololobovo [8] & $\mathrm{RU}$ & 55.05 & 38.57 & 272.00 & 68.00 & 0.25 \\
\hline Gorokhov [5] & UA & 50.31 & 24.50 & 435.00 & 108.75 & 0.25 \\
\hline Grubgraben Kammern & $\mathrm{AT}$ & 48.50 & 15.80 & 2100.00 & 0.00 & 0 \\
\hline \multicolumn{7}{|l|}{ Niederösterreich [2] } \\
\hline Gunderding Oberösterreich [2] & AT & 48.26 & 13.23 & 10340.00 & 0.00 & 0 \\
\hline Halytsch (Halyc) [9] & UA & 49.10 & 24.80 & 226.00 & 56.50 & 0.25 \\
\hline Harmignies [10] & $\mathrm{BE}$ & 50.41 & 4.02 & 412.00 & 103.00 & 0.25 \\
\hline Harmignies [2] & $\mathrm{BE}$ & 50.41 & 4.02 & 1467.00 & 0.00 & 0 \\
\hline Harmignies [2] & $\mathrm{BE}$ & 50.41 & 4.02 & 3135.00 & 0.00 & 0 \\
\hline Irig [4] & RS & 45.08 & 19.87 & 192.00 & 44.00 & 0.23 \\
\hline Katymár [7] & $\mathrm{HU}$ & 46.02 & 19.20 & 1005 & 0.00 & 0 \\
\hline Katymár [4] & $\mathrm{HU}$ & 46.02 & 19.20 & 632.00 & 144.00 & 0.23 \\
\hline Kesselt [11] & $\mathrm{BE}$ & 50.84 & 5.60 & 446.00 & 111.50 & 0.25 \\
\hline
\end{tabular}


Table S1: continued

\begin{tabular}{|c|c|c|c|c|c|c|}
\hline Site & $\mathrm{CC}$ & $\begin{array}{l}\text { Lat } \\
{ }^{\circ} \mathrm{N}\end{array}$ & $\begin{array}{r}\text { Lon } \\
{ }^{\circ} \mathrm{E}\end{array}$ & $\begin{array}{r}\text { MAR } \\
\mathrm{g} \mathrm{m}^{-2} \mathrm{yr}^{-1}\end{array}$ & $\begin{array}{l}\text { MAR10 } \\
\mathrm{g} \mathrm{m}^{-2} \mathrm{yr}^{-1}\end{array}$ & Frac10 \\
\hline Kesselt [2] & $\mathrm{BE}$ & 50.84 & 5.60 & 825.00 & 0.00 & 0 \\
\hline Kesselt [2] & $\mathrm{BE}$ & 50.84 & 5.60 & 330.00 & 0.00 & 0 \\
\hline Korostelevo (Korostylievo) [12] & RU & 51.84 & 42.42 & 181.00 & 45.25 & 0.25 \\
\hline Lakitelek 1 [4] & $\mathrm{HU}$ & 46.88 & 20.02 & 254.00 & 58.00 & 0.23 \\
\hline Látókép [4] & $\mathrm{HU}$ & 47.56 & 21.49 & 212.00 & 48.00 & 0.23 \\
\hline Likhvin (Chekalin) [8] & $\mathrm{RU}$ & 54.10 & 36.27 & 272.00 & 68.00 & 0.25 \\
\hline Madaras [4] & $\mathrm{HU}$ & 46.04 & 19.29 & 375.00 & 86.00 & 0.23 \\
\hline Mende [13] & $\mathrm{HU}$ & 47.42 & 19.45 & 456.00 & 114.00 & 0.25 \\
\hline Mende [2] & $\mathrm{HU}$ & 47.42 & 19.45 & 519.00 & 0.00 & 0 \\
\hline Mende [4] & $\mathrm{HU}$ & 47.42 & 19.45 & 761.00 & 173.00 & 0.23 \\
\hline Mezyn (Mezin) [5] & UA & 52.20 & 33.30 & 788.00 & 197.00 & 0.25 \\
\hline Molodova V [14] & UA & 48.50 & 26.89 & 369.00 & 92.25 & 0.25 \\
\hline Mosorin [7] & RS & 45.26 & 20.28 & 545.00 & 0.00 & 0 \\
\hline Mošorin [4] & RS & 45.26 & 20.28 & 395.00 & 90.00 & 0.23 \\
\hline Nussloch [15] & $\mathrm{DE}$ & 49.35 & 8.72 & 2114.00 & 528.50 & 0.25 \\
\hline Nussloch [2] & $\mathrm{DE}$ & 49.35 & 8.72 & 1213.00 & 0.00 & 0 \\
\hline Nussloch [2] & $\mathrm{DE}$ & 49.35 & 8.72 & 6129.00 & 0.00 & 0 \\
\hline Otkaznoe (Otkaznoye) [16] & RU & 44.32 & 43.85 & 336.00 & 117.60 & 0.35 \\
\hline Paks [13] & $\mathrm{HU}$ & 46.64 & 18.88 & 1325.00 & 331.25 & 0.25 \\
\hline Paks [2] & $\mathrm{HU}$ & 46.64 & 18.88 & 2662.00 & 0.00 & 0 \\
\hline Paks [4] & $\mathrm{HU}$ & 46.64 & 18.88 & 1422.00 & 324.00 & 0.23 \\
\hline Patkóbánya Kopasz Hill Tokaj [17] & $\mathrm{HU}$ & 48.22 & 20.45 & 395.00 & 98.75 & 0.25 \\
\hline Petrovaradin [4] & RS & 45.27 & 19.87 & 174.00 & 40.00 & 0.23 \\
\hline Prymors'ke (Primorskoje) [18] & UA & 45.94 & 30.20 & 654.00 & 163.50 & 0.25 \\
\hline Pyrogove [7] & UA & 50.36 & 30.53 & 1659.00 & 0.00 & 0 \\
\hline Radymno [7] & $\mathrm{PL}$ & 49.96 & 22.81 & 538.00 & 0.00 & 0 \\
\hline Remicourt [2] & $\mathrm{BE}$ & 50.67 & 5.40 & 560.00 & 140.00 & 0.25 \\
\hline Remicourt [2] & $\mathrm{BE}$ & 50.67 & 5.40 & 453.00 & 0.00 & 0 \\
\hline Rocourt [19] & $\mathrm{BE}$ & 50.68 & 5.54 & 257.00 & 64.25 & 0.25 \\
\hline Rocourt [2] & $\mathrm{BE}$ & 50.68 & 5.54 & 93.00 & 0.00 & 0 \\
\hline Ruma [4] & RS & 45.01 & 19.85 & 192.00 & 44.00 & 0.23 \\
\hline Sables d'Or les Pins [2] & FR & 48.65 & -2.39 & 354.00 & 0.00 & 0 \\
\hline Ságvár [4] & $\mathrm{HU}$ & 46.83 & 18.09 & 176.00 & 40.00 & 0.23 \\
\hline Sanzhijka [7] & UA & 46.23 & 30.61 & 808.00 & 0.00 & 0 \\
\hline Schwalbenberg [2] & $\mathrm{DE}$ & 50.57 & 7.24 & 560.00 & 140.00 & 0.25 \\
\hline St.-Pierre-les-Elbeuf [20] & FR & 49.60 & 1.23 & 242.00 & 60.50 & 0.25 \\
\hline St.-Romain-de-Colbosc [19] & FR & 49.54 & 0.36 & 687.00 & 171.75 & 0.25 \\
\hline Stari Bezradychy [7] & UA & 50.18 & 30.55 & 440.00 & 0.00 & 0 \\
\hline Stari Slankamen [4] & RS & 45.13 & 20.27 & 168.00 & 38.00 & 0.23 \\
\hline $\begin{array}{l}\text { Stillfried Gänserndorf } \\
\text { Niederösterreich [2] }\end{array}$ & AT & 48.42 & 16.84 & 229.00 & 0.00 & 0 \\
\hline Strelitsa [21] & $\mathrm{RU}$ & 51.60 & 38.90 & 290.00 & 72.50 & 0.25 \\
\hline Surduk [4] & RS & 45.07 & 20.33 & 434.00 & 99.00 & 0.23 \\
\hline Susek [4] & RS & 45.22 & 19.53 & 150.00 & 34.00 & 0.23 \\
\hline Süttő[22] & $\mathrm{HU}$ & 47.74 & 18.45 & 1009.00 & 0.00 & 0 \\
\hline
\end{tabular}


Table S1: continued

\begin{tabular}{llrrrrr} 
Site & CC & $\begin{array}{r}\text { Lat } \\
{ }^{\circ} \mathrm{N}\end{array}$ & $\begin{array}{r}\text { Lon } \\
{ }^{\circ} \mathrm{E}\end{array}$ & $\begin{array}{r}\text { MAR } \\
\mathrm{g} \mathrm{m}^{-2} \mathrm{yr}^{-1}\end{array}$ & $\begin{array}{r}\text { MAR10 } \\
\mathrm{g} \mathrm{m}^{-2} \mathrm{yr}^{-1}\end{array}$ & Frac10 \\
\hline Süttö[4] & HU & 47.74 & 18.45 & 584.00 & 133.00 & 0.23 \\
Szeged-Öthalom I [4] & HU & 46.28 & 20.10 & 332.00 & 76.00 & 0.23 \\
Tápiósüly [4] & HU & 47.45 & 19.52 & 504.00 & 115.00 & 0.23 \\
Titel [7] & RS & 45.23 & 20.30 & 591.00 & 0.00 & 0 \\
Titel [4] & RS & 45.23 & 20.30 & 510.00 & 116.00 & 0.23 \\
Tokaj (Kereszt Hill II) [4] & HU & 48.13 & 21.40 & 222.00 & 51.00 & 0.23 \\
Tokaj (Patkó-quarry) [4] & HU & 48.12 & 21.40 & 332.00 & 76.00 & 0.23 \\
Tokaj Kopasz Hill Patkó-bánya [17] & HU & 48.22 & 20.45 & 395.00 & 98.75 & 0.25 \\
Tönchesberg Tönches-Berg Kruft [23] & DE & 50.35 & 7.35 & 779.00 & 194.75 & 0.25 \\
Tönchesberg Tönches-Berg Kruft [2] & DE & 50.35 & 7.35 & 1249.00 & 0.00 & 0 \\
Trindorf Oftering [2] & AT & 48.24 & 14.14 & 2970.00 & 0.00 & 0 \\
Üveghuta-2 borehole [4] & HU & 46.20 & 18.61 & 338.00 & 77.00 & 0.23 \\
Volgodonsk [24] & RU & 47.56 & 41.99 & 245.00 & 98.00 & 0.4 \\
Vyazivok [25] & UA & 49.33 & 32.98 & 202.00 & 50.50 & 0.25 \\
Willendorf II Niederösterreich [2] & AT & 47.79 & 16.05 & 372.00 & 0.00 & 0 \\
Willendorf II Niederösterreich [2] & AT & 47.79 & 16.05 & 886.00 & 0.00 & 0 \\
Zmajevac [4] & HR & 45.81 & 18.82 & 437.00 & 100.00 & 0.23
\end{tabular}


Table S2: Palaeoclimate Modelling Intercomparison Project Phase 3 (PMIP3) setup for global LGM simulations (first column) and its adapted implementation that upgrades the WRF-Chem to the WRFChem-LGM. Default values listed for comparison to the right of the slash (second column).

\begin{tabular}{|c|c|c|}
\hline & PMIP3-LGM [26] & WRF-Chem-LGM / WRF-Chem \\
\hline \multicolumn{3}{|l|}{ Earth's orbit: } \\
\hline Eccentricity & 0.018994 & $0.018994 / 0.014$ \\
\hline Obliquity & $22.949^{\circ}$ & $22.949^{\circ} / 23.5^{\circ}$ \\
\hline \multicolumn{3}{|l|}{ Gas concentrations: } \\
\hline $\mathrm{CO}_{2}\left(10^{-6}\right)$ & 185 & $185 / 379$ \\
\hline $\mathrm{CH}_{4}\left(10^{-9}\right)$ & 350 & $350 / 1774$ \\
\hline $\mathrm{N}_{2} \mathrm{O}\left(10^{-9}\right)$ & 200 & $200 / 319$ \\
\hline CFCs, misc. $\left(10^{-12}\right)$ & 0 & $0 / 169,251,538$ \\
\hline Mineral dust & Computed or CMIP5-PI [27] & Shao et al. [28] \\
\hline Land-sea mask & $1^{\circ}$ PMIP3-LGM [26] & $1^{\circ}$ PMIP3-LGM-based \\
\hline Orography & Offsets added (1 PMIP3-LGM) & Offsets added ( $1^{\circ}$ PMIP3-LGM-based) \\
\hline Ice sheets & $1^{\circ} \mathrm{PMIP} 3-\mathrm{LGM}$ & $2^{\circ}$ CLIMAP-LGM [29]-based \\
\hline Land use & Same as in CMIP5-PI & $2^{\circ}$ CLIMAP-LGM-based \\
\hline Vegetation cover & Same as in CMIP5-PI & $\begin{array}{l}\text { Deduced from } 2^{\circ} \text { CLIMAP-LGM and } \\
\text { WRF geo-data [30] }\end{array}$ \\
\hline Soil types & Not specified & $\begin{array}{l}\text { Present-day WRF geo-data, EIS } \\
\text { adapted }\end{array}$ \\
\hline Erodibility & Not specified & $\begin{array}{l}\text { Deduced [31] from } 1^{\circ} \text { PMIP3-LGM } \\
\text { topography and } 2^{\circ} \text { CLIMAP-LGM } \\
\text { bare soil }\end{array}$ \\
\hline Sea surface temperatures & Not specified & MPI-LGM [32-34] \\
\hline
\end{tabular}

Table S3: Modules and domain parameters applied to run the WRF-Chem-LGM simulations. University of Cologne (UC)

\begin{tabular}{ll}
\hline WRF-Chem version & 3.5 .1 \\
Time step & $3 \mathrm{~min}$ \\
Horizontal resolution & $50 \mathrm{~km}$ \\
Vertical levels & 35 \\
MPI-LGM boundary data input interval & $6 \mathrm{~h}$ \\
Microphysics & Lin Scheme [35] \\
Longwave, Shortwave Radiation & RRTMG [36] \\
Surface Layer & MM5 Similarity Scheme [37] \\
Land Surface Model & Unified Noah [38-40] \\
Planetary Boundary layer & Yonsei Univ. Scheme [41] \\
Cumulus convection parameter & Tiedtke Scheme [42] \\
Non hydrostatic & Yes \\
Chemistry modules active & Dust-only \\
Dry deposition & Yes [43] \\
Vertical turbulent mixing & Yes \\
Dust option & GOCART [44] \\
Dust emissions & UC Simplified Scheme [28] \\
Wet deposition & Enabled [45] \\
\hline
\end{tabular}




\section{References}

[1] Rousseau DD, Zöller L, Valet JP (1998) Late Pleistocene Climatic Variations at Achenheim, France, Based on a Magnetic Susceptibility and TL Chronology of Loess. Quaternary Research 49(3):255-263.

[2] Frechen M (2003) Loess in Europe-mass accumulation rates during the Last Glacial Period. Quaternary Science Reviews 22(18-19):1835-1857.

[3] Novothny A, Horváth E, Frechen M (2002) The loess profile at Albertirsa, Hungaryimprovements in loess stratigraphy by luminescence dating. Quaternary International 9596:155-163.

[4] Újvári G, Kovács J, Varga G, Raucsik B, Marković SB (2010) Dust flux estimates for the Last Glacial Period in East Central Europe based on terrestrial records of loess deposits: a review. Quaternary Science Reviews 29(23-24):3157-3166.

[5] Mahowald NM, et al. (2006) Change in atmospheric mineral aerosols in response to climate: Last glacial period, preindustrial, modern, and doubled carbon dioxide climates. F. of Geophysical Research: Atmospheres 111(D10).

[6] Frechen M, Zander A, Cílek V, Ložek V (1999) Loess chronology of the Last Interglacial/Glacial cycle in Bohemia and Moravia, Czech Republic. Quaternary Science Reviews 18(13):1467-1493.

[7] Bokhorst M, et al. (2011) Atmospheric circulation patterns in central and eastern Europe during the Weichselian Pleniglacial inferred from loess grain-size records. Quaternary International 234(1-2):62-74.

[8] Little EC, et al. (2002) Quaternary stratigraphy and optical dating of loess from the east European Plain (Russia). Quaternary Science Reviews 21(14-15):1745-1762.

[9] Eanczont M, Madeyska T (2005) Environment of the East Carpathian Foreland during periods of Palaeolithic man's activity. CATENA 59(3):319-340.

[10] Frechen M, van Vliet-Lanoë B, van den Haute P (2001) The Upper Pleistocene loess record at Harmignies/Belgium - high resolution terrestrial archive of climate forcing. Palaeogeography, Palaeoclimatology, Palaeoecology 173(3-4):175-195.

[11] van den Haute P, Vancraeynest L, De Corte F (1998) The Late Pleistocene loess deposits and palaeosols of eastern Belgium: new TL age determinations. F. of Quaternary Science 13(5):487497.

[12] Rutter NW, et al. (2003) Correlation and interpretation of paleosols and loess across European Russia and Asia over the last interglacial-glacial cycle. Quaternary Research 60(1):101-109.

[13] Frechen M, Horváth E, Gábris G (1997) Geochronology of Middle and Upper Pleistocene Loess Sections in Hungary. Quaternary Research 48(3):291-312.

[14] Haesaerts P, et al. (2003) The east Carpathian loess record: a reference for the middle and late pleniglacial stratigraphy in central Europe [La séquence loessique du domaine est-carpatique : une référence pour le Pléniglaciaire moyen et supérieur d'Europe centrale.]. Quaternaire 14(3):163-188.

[15] Rousseau D, et al. (2002) Abrupt millennial climatic changes from Nussloch (Germany) Upper Weichselian eolian records during the Last Glaciation. Quaternary Science Reviews 21(1415):1577-1582. 
[16] Molodkov AN, Bolikhovskaya NS (2002) Eustatic sea-level and climate changes over the last $600 \mathrm{ka}$ as derived from mollusc-based ESR-chronostratigraphy and pollen evidence in Northern Eurasia. Sedimentary Geology 150(1-2):185-201.

[17] Sümegi P, Rudner ZE (2001) In situ charcoal fragments as remains of natural wild fires in the upper Würm of the Carpathian Basin. Quaternary International 76-77:165-176.

[18] Nawrocki J, Bakhmutov V, Bogucki A, Dolecki L (1999) The paleo- and petromagnetic record in the Polish and Ukrainian loess-paleosol sequences. Physics and Chemistry of the Earth, Part A: Solid Earth and Geodesy 24(9):773-777.

[19] Wintle AG (1987) Thermoluminescence dating of loess at Rocourt, Belgium. Geologie en Mijnbouw 66(1):35-42.

[20] Antoine P, Rousseau DD, Lautridou JP, Hatté C (1999) Last interglacial-glacial climatic cycle in loess-palaeosol successions of north-western France. Boreas 28(4):551-563.

[21] Virina EI, et al. (2000) Palaeoclimatic record in the loess-palaeosol sequence of the Strelitsa type section (Don glaciation area, Russia) deduced from rock magnetic and palynological data. f. of Quaternary Science 15(5):487-499.

[22] Novothny A, Frechen M, Horváth E, Wacha L, Rolf C (2011) Investigating the penultimate and last glacial cycles of the Süttő loess section (Hungary) using luminescence dating, highresolution grain size, and magnetic susceptibility data. Quaternary International 234(1-2):7585.

[23] Frechen M (1992) Systematic thermoluminescence dating of two loess profiles from the Middle Rhine Area (F.R.G.). Quaternary Science Reviews 11(1-2):93-101.

[24] Trofimov VT, ed. (2001) Loess mantle of the Earth, and its properties. (Moscow University Press).

[25] Rousseau DD, Gerasimenko N, Matviischina Z, Kukla G (2001) Late Pleistocene Environments of the Central Ukraine. Quaternary Research 56(3):349-356.

[26] Braconnot P, et al. (2012) Evaluation of climate models using palaeoclimatic data. Nature Climate Change 2(6):417-424.

[27] Taylor KE, Stouffer RJ, Meehl GA (2012) An Overview of CMIP5 and the Experiment Design. Bull. of the American Meteorological Society 93(4):485-498.

[28] Shao Y (2004) Simplification of a dust emission scheme and comparison with data. F. of Geophysical Research 109(D10).

[29] Climate, Long-Range Investigation, Mapping and Prediction (CLIMAP) Project Members, Ruddiman WF (1984) The Last Interglacial Ocean. Quaternary Research 21(2):123-224.

[30] Grell GA, et al. (2005) Fully coupled "online" chemistry within the WRF model. Atmospheric Environment 39(37):6957-6975.

[31] Ginoux P, et al. (2001) Sources and distributions of dust aerosols simulated with the GOCART model. F. of Geophysical Research: Atmospheres 106(D17):20255-20273.

[32] Stevens B, et al. (2013) Atmospheric component of the MPI-M Earth System Model: ECHAM6. f. of Advances in Modeling Earth Systems 5:146-172. 
[33] Jungclaus JH, et al. (2013) Characteristics of the ocean simulations in the Max Planck Institute Ocean Model (MPIOM) the ocean component of the MPI-Earth system model. F. of Advances in Modeling Earth Systems 5(2):422-446.

[34] Jungclaus J, et al. (2012) CMIP5 simulations of the Max Planck Institute for Meteorology (MPIM) based on the MPI-ESM-P model: The lgm experiment, served by ESGF (WDCC at DKRZ).

[35] Lin Y, Farley R, Orville H (1983) Bulk Parameterization of the Snow Field in a Cloud Model. f. of Applied Meteorology and Climatology 22:1065-1092.

[36] Iacono MJ, et al. (2008) Radiative forcing by long-lived greenhouse gases: Calculations with the AER radiative transfer models. F. of Geophysical Research 113(D13).

[37] Webb EK (1970) Profile relationships: The log-linear range, and extension to strong stability. Quarterly 7. of the Royal Meteorological Society 96:67-90.

[38] Rosero E, Yang ZL, Gulden LE, Niu GY, Gochis DJ (2009) Evaluating Enhanced Hydrological Representations in Noah LSM over Transition Zones: Implications for Model Development. 7. of Hydrometeorology 10(3):600-622.

[39] Case JL, Crosson WL, Kumar SV, Lapenta WM, Peters-Lidard CD (2008) Impacts of HighResolution Land Surface Initialization on Regional Sensible Weather Forecasts from the WRF Model. F. of Hydrometeorology 9(6):1249-1266.

[40] Tewari M, et al. (2004) Implementation and verification of the unified NOAH land surface model in the WRF model. 20th conference on weather analysis and forecasting/16th conference on numerical weather prediction 1115:11-15.

[41] Hong SY, Noh Y, Dudhia J (2006) A New Vertical Diffusion Package with an Explicit Treatment of Entrainment Processes. Monthly Weather Review 134(9):2318-2341.

[42] Zhang C, Wang Y, Hamilton K (2011) Improved Representation of Boundary Layer Clouds over the Southeast Pacific in ARW-WRF Using a Modified Tiedtke Cumulus Parameterization Scheme. Monthly Weather Review 139(11):3489-3513.

[43] Wesely M (1989) Parameterization of surface resistances to gaseous dry deposition in regionalscale numerical models. Atmospheric Environment (1967) 23(6):1293-1304.

[44] Chin M, Rood RB, Lin SJ, Müller JF, Thompson AM (2000) Atmospheric sulfur cycle simulated in the global model GOCART: Model description and global properties. F. of Geophysical Research: Atmospheres 105(D20):24671-24687.

[45] Jung E, Shao Y, Sakai T (2005) A study on the effects of convective transport on regional-scale Asian dust storms in 2002. F. of Geophysical Research: Atmospheres 110(D20). D20201. 